\title{
Quantitative mapping of collagen fiber alignment in thick tissue samples using transmission polarized- light microscopy
}

Dmitry D. Yakovlev

Marina E. Shvachkina

Maria M. Sherman

Andrey V. Spivak

Alexander B. Pravdin

Dmitry A. Yakovlev 


\title{
Quantitative mapping of collagen fiber alignment in thick tissue samples using transmission polarized-light microscopy
}

\author{
Dmitry D. Yakovlev, Marina E. Shvachkina, Maria M. Sherman, Andrey V. Spivak, Alexander B. Pravdin, and \\ Dmitry A. Yakovlev* \\ Saratov State University, Faculty of Physics, Department of Optics and Biophotonics, Astrakhanskaya 83, Saratov 410012, Russia
}

\begin{abstract}
Immersion optical clearing makes it possible to use transmission polarized-light microscopy for characterization of thick (200 to $2000 \mu \mathrm{m}$ ) layers of biological tissues. We discuss polarization properties of thick samples in the context of the problem of characterization of collagen fiber alignment in connective tissues such as sclera and dermis. Optical chirality caused by azimuthal variations of the macroscopic (effective) optic axis of the medium across the sample thickness should be considered in polarization mapping of thick samples of these tissues. We experimentally evaluate to what extent the optical chirality affects the measurement results in typical situations and show under what conditions it can be easily taken into account and does not hinder, but rather helps, in characterization of collagen fiber alignment. ๑ 2016 Society of Photo-Optical Instrumentation Engineers (SPIE) [DOI: 10.1117/1.JBO.21.7.071111]
\end{abstract}

Keywords: collagen fiber alignment; polarization mapping; microscopy; sclera; dermis.

Paper 150737SSRR received Oct. 31, 2015; accepted for publication Mar. 4, 2016; published online Mar. 30, 2016.

\section{Introduction}

Many biological tissues exhibit birefringence. Examples of tissues with birefringent components include muscle, bone, tendon, cartilage, stratum corneum, dermis, cornea, sclera, dura mater, blood vessel walls, nervous tissue elements (myelin), dentine, and so on. ${ }^{1,2}$ The birefringence of biological tissue is always an indicator of the orientational order of its structural elements at the level of molecules and supramolecular aggregates. The birefringence of components of biological tissues gives an opportunity to use optical polarization methods for tissue characterization. Transmission polarized-light microscopy (TPM) has the longest history of application to biological tissues. For many decades, it was used to study biotissue structure, characterize tissue physiological state, and perform monitoring of biological processes. ${ }^{2-27}$ Nowadays, TPM continues to be widely applied in biology and medicine, along with other effective and diverse polarization methods ${ }^{28-49}$ (polarization-sensitive optical coherence tomography, ${ }^{28-34}$ macroscopic Mueller matrix imaging, ${ }^{35-42}$ polarization spectroscopy, ${ }^{43-45}$ and so on). Modern methods of polarized-light microscopy exploit digital imaging and elaborate data processing. One of the most advanced methods in this regard is the microscopic Mueller matrix imaging. ${ }^{3-6}$ High-numerical aperture (NA) TPM is used to visualize cellular and extracellular elements of micron and submicron size $\mathrm{s}^{3-5,7-16}$ and works with the very thin samples. Low-NA TPM is used for characterization of tissues at the macrolevel. ${ }^{17-21}$ Sample thickness for low-NA TPM is usually about $30 \mu \mathrm{m}$.

One of the tasks accomplished with the aid of low-NA polarized-light microscopy is generating maps of collagen fiber alignment in connective tissues such as the sclera and dermis. ${ }^{3,17,18}$ Such maps are required, in particular, in solving biomechanical problems, ${ }^{17,50}$ since the alignment of collagen fibers in tissues substantially affects their mechanical properties. Of interest in such cases are the direction of preferred orientation of fibers and the degree of orientational order. The applicability of polarization methods to this problem stems from the birefringence of collagen fibers. Practice shows that for strongly scattering fibrous tissues, which are the dermis and sclera in their native state, TPM mapping is applicable to samples whose thickness does not exceed $120 \mu \mathrm{m}$; with a greater thickness of the sample, reliable results cannot be obtained due to the strong multiple scattering. Usually, information about collagen fiber alignment is required for the tissue as a whole, when the thickness of the tissue is from 400 to $2000 \mu \mathrm{m}$. The need for sectioning the tissue makes the measurement procedure very laborious. Furthermore, in making sections the orientation of collagen fibers may be disturbed. Therefore, there is a need for more efficient and reliable methods of determining orientational parameters of the collagen fiber network. In this paper, through the sclera and dermis examples, we demonstrate that the TPM mapping of thick samples may be successfully performed using the technique of immersion optical clearing of tissues. Impregnation of tissues such as stroma of sclera and dermis by an optical clearing agent such as glycerol significantly reduces the scattering of light within the tissue and, due to this, substantially increases its transparency. ${ }^{1}$ One of the factors that should be taken into account in polarization mapping of thick samples is an azimuthal variation of the macroscopic local optic axis across the sample thickness. In this work, we demonstrate that in many cases, in studying collagen fiber alignment in thick tissue samples, such variation can be easily taken into account. The analysis and estimates presented here may be useful in considering not only the sclera and dermis, 
but also other collagen-containing tissues, e.g., heart valve leaflets.

\section{Collagen Structure and the Birefringence of Sclera and Dermis}

The sclera is a dense connective tissue which mainly consists of collagen fibrils, 25 to $230 \mathrm{~nm}$ in diameter, packed in tape-shaped bundles forming a layered structure..$^{51,52}$ The bundle width is 1 to $50 \mu \mathrm{m}$ and thickness is 0.5 to $6 \mu \mathrm{m}$. The bundles go in various directions, but are always oriented parallel to the surface of sclera. The bundles may be separated by large cavities filled with interstitial fluid (i.e., the packing density of collagen bundles in different regions of sclera may differ). The collagen network organization varies from region to region and is different in the inner and outer layers of sclera. ${ }^{51,52}$ The scleral stroma contains relatively few cellular elements and vessels. The dermis contains more such inclusions. The diameter of collagen bundles in dermis is $\sim 1$ to $8 \mu \mathrm{m} .{ }^{53}$ In the dermis, there is no strict stratification of collagen structure, which is characteristic of the sclera. Some of the collagen bundles are not parallel to the surface of the skin. Nevertheless, the local preferred directions of the collagen bundle orientation are parallel to that surface. ${ }^{9,54}$ The direction of the preferred orientation of collagen fibers in sclera and dermis, being parallel to the surface, can vary significantly over the distances of the order of hundreds of microns. ${ }^{51,52}$ Significant spatial variations in the degree of orientational order of collagen fibers are also observed. ${ }^{50}$

The collagen fiber, as a system of orientationally ordered fibrils, can be considered as a basic birefringent element of the tissue. Collagen fiber exhibits positive birefringence and has its optic axis directed along its longitudinal axis. In this study, we deal with macroscopic local characteristics of tissue birefringence-a macroscopic optic axis (we indicate its direction with a unit vector c) and macroscopic principal refractive indices $\left(n_{\|}\right.$and $n_{\perp}$ )—which by definition are associated with relatively large elementary volumes, with dimensions of the order of tens of microns, through which a large number of differently oriented collagen fibers run. The use of these macroscopic parameters leads to the consideration of the collagen bundles-interstitial fluid system as a continuous optically anisotropic medium. In experimental assessments of birefringence parameters of collagenous tissues, this model is used in most cases, implicitly or explicitly. The use of this model can be reasonable in situations where multiple light scattering by collagen fibers can be neglected.

The direction of the macroscopic optic axis associated with an elementary volume in the tissue is determined by the direction of preferred orientation of collagen fibers within this volume, and the value of the index of birefringence $\Delta n=n_{\|}-n_{\perp}$ depends on the degree of orientational order of the collagen fibers there. The latter allows estimating the degree of orientational order of the fibers by experimental values of $\Delta n$ (Ref. 55). The magnitude of $\Delta n$ is proportional to the volume fraction of collagen fibers in the tissue. Assessed values of $\Delta n$ for tissues with dense packing and high orientational order of collagen fibers (native state) are $3 \times 10^{-3}$ for cat corneal lamella ${ }^{20}$ and $(3 \pm 0.6) \times 10^{-3}$ for rat tail tendon. ${ }^{27}$

Under immersion clearing of native collagenous tissues, the value of $\Delta n$ greatly reduces. This is explained by a decrease in the contribution of form anisotropy to the total birefringence. With pure glycerol as a clearing agent, this effect is maximal: $\Delta n$ may decrease by a factor of 2 to 3 (Refs. 20 and 56).
Based on the preceding information on the collagen fiber alignment in sclera and dermis, in an analysis of results of low-NA polarization mapping, we can take as a model of the collagen matrix in a cleared sclera or dermis sample a layer of locally uniaxial medium with spatial distribution of the local optic axis of the form

$\mathbf{c}=\left(\begin{array}{c}c_{x} \\ c_{y} \\ c_{z}\end{array}\right)=\left(\begin{array}{c}\cos \varphi(x, y, z) \\ \sin \varphi(x, y, z) \\ 0\end{array}\right)$,

where $\varphi$ is the angle between the optic axis and the $x$-axis of a coordinate system $(x, y, z)$ with the $z$-axis directed perpendicular to the tissue surface. When considering thick layers, we have to take into account that $\mathbf{c}$ varies with $z$. Our main goal here is to assess whether we can with the help of low-NA polarization mapping estimate with good accuracy the parameters of collagen fiber alignment in thick layers of dermis and sclera taking into account that $\mathbf{c}$ depends on $z$.

\section{Optical Characteristics Being Mapped}

We assume the following conditions of examination of tissue samples: a sample of well-cleared tissue is placed in immersion fluid between an object-plate and a cover glass and observed with a polarizing microscope, the sample being illuminated with an almost parallel beam, and a microscope objective with small numerical aperture being used. Images of the sample in quasimonochromatic light are acquired with a digital camera.

We assume that the local measured transmission of the sample can be described by a Mueller matrix $\mathbf{M}=$ $\left[m_{i j}\right] \quad(i, j=1,2,3,4)$ linking the Stokes vector of the light incident on the sample and the Stokes vector of the component of the light emerging from a given small region of the sample that enters the microscope objective and is involved in the formation of images of this region. We denote this component by $\mathcal{W}$. When the measurements are carried out without the use of phase plates, with a linear polarizer and a linear analyzer only, in order to describe how the recorded intensity depends on the orientation of the polarizer and analyzer it is convenient to use the concept of polarization transfer factor ${ }^{57,58}$ which in this case can be defined as follows. Let $\vartheta$ and $\vartheta^{\prime}$ be the angles of orientation of the transmission axes of the polarizer and analyzer, respectively, measured from the $x$-axis of a coordinate system $(x, y, z)$ [see Figs. 1(a) and 1(b)], and let $\bar{\vartheta}$ and $\bar{\vartheta}^{\prime}$ be arbitrary values of $\vartheta$ and $\vartheta^{\prime}$. We define the polarization transfer factor of the given small region (domain) of the sample for $\vartheta=\bar{\vartheta}$ and $\vartheta^{\prime}=\bar{\vartheta}^{\prime}$ as

$t_{P A}\left(\bar{\vartheta}, \bar{\vartheta}^{\prime}\right)=I_{\mathcal{W} \bar{\vartheta}^{\prime}}(\bar{\vartheta}) / I_{\mathrm{inc}}$,

where $I_{\mathcal{W} \bar{\vartheta}^{\prime}}(\bar{\vartheta})$ is the intensity of the linearly polarized, with vibration direction at angle $\bar{\vartheta}^{\prime}$ with respect to the $x$-axis, component of $\mathcal{W}$ when $\vartheta=\bar{\vartheta} ; I_{\text {inc }}$ is the intensity of the incident light. The dependence of $t_{P A}$ on $\vartheta$ and $\vartheta^{\prime}$ can be expressed as follows:

$$
\begin{aligned}
t_{P A}\left(\vartheta, \vartheta^{\prime}\right)= & \tilde{B}_{0}+\tilde{B}_{1} \cos \eta^{-}+\tilde{B}_{2} \cos \eta^{+}+\tilde{B}_{3} \sin \eta^{-} \\
& +\tilde{B}_{4} \sin \eta^{+}+\tilde{B}_{5} \cos 2 \vartheta+\tilde{B}_{6} \sin 2 \vartheta \\
& +\tilde{B}_{7} \cos 2 \vartheta^{\prime}+\tilde{B}_{8} \sin 2 \vartheta^{\prime}
\end{aligned}
$$

$\eta^{-}=2\left(\vartheta-\vartheta^{\prime}\right), \quad \eta^{+}=2\left(\vartheta+\vartheta^{\prime}\right)$, 


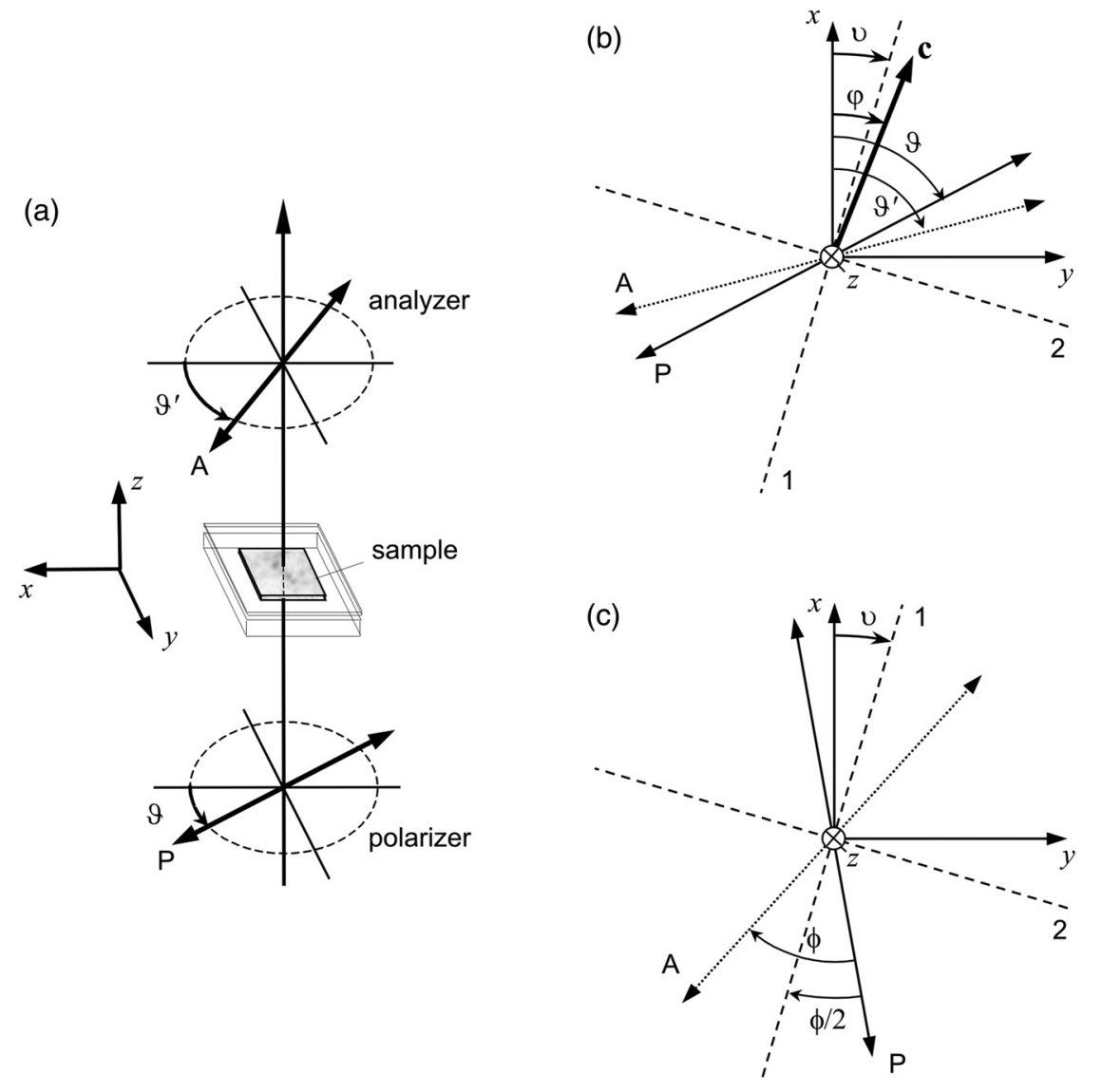

Fig. 1 (a) Geometry of the experiment. $P$ and $A$ are the transmission axes of the polarizer and analyzer, respectively. (b) Specification of orientation of the polarizer, analyzer, polarization midaxes (1 and 2), and optic axis c. (c) Orientation of the polarizer and analyzer corresponding to the brightest appearance of the domain.

where

$$
\begin{aligned}
& \tilde{B}_{0}=m_{11} / 2, \\
& \tilde{B}_{1}=\left(m_{22}+m_{33}\right) / 4, \\
& \tilde{B}_{2}=\left(m_{22}-m_{33}\right) / 4, \\
& \tilde{B}_{3}=\left(m_{23}-m_{32}\right) / 4, \\
& \tilde{B}_{4}=\left(m_{23}+m_{32}\right) / 4, \\
& \tilde{B}_{5}=m_{12} / 2, \quad \tilde{B}_{6}=m_{13} / 2, \\
& \tilde{B}_{7}=m_{21} / 2, \quad \tilde{B}_{8}=m_{31} / 2 .
\end{aligned}
$$

Regarding the coefficients $\tilde{B}_{j}$, note the following. The ratio of the intensity of $\mathcal{W}$ to the intensity of the incident light would be equal to $2 \tilde{B}_{0}$ if the incident light were unpolarized. In the absence of natural optical activity (in what follows, we neglect the natural optical activity of the constituents of tissues since it has insignificant effect on the measured quantities in the situation at hand; see estimates in Ref. 59), for optically isotropic media, the coefficients $\tilde{B}_{2}-\tilde{B}_{8}$ are equal to zero. If the intensity of $\mathcal{W}$ is independent of the state of polarization of the incident light (i.e., when there are no polarization-dependent losses), the coefficients $\tilde{B}_{5}-\tilde{B}_{8}$ are equal to zero. In our mapping experiments with glycerol-cleared sclera and dermis, we have not met any noticeable manifestations of polarization-dependent losses in these tissues. In the absence of polarization-dependent losses,
Eq. (3) is simplified to

$$
\begin{aligned}
t_{P A}\left(\vartheta, \vartheta^{\prime}\right)= & \tilde{B}_{0}+\tilde{B}_{1} \cos 2\left(\vartheta-\vartheta^{\prime}\right)+\tilde{B}_{2} \cos 2\left(\vartheta+\vartheta^{\prime}\right) \\
& +\tilde{B}_{3} \sin 2\left(\vartheta-\vartheta^{\prime}\right)+\tilde{B}_{4} \sin 2\left(\vartheta+\vartheta^{\prime}\right)
\end{aligned}
$$

In this study, of special interest for us are three characteristic angles, $v, \phi$, and $\Delta_{r}$, defined by the relations

$\cos 4 v=\tilde{B}_{2} / \sqrt{\tilde{B}_{2}^{2}+\tilde{B}_{4}^{2}}, \quad \sin 4 v=\tilde{B}_{4} / \sqrt{\tilde{B}_{2}^{2}+\tilde{B}_{4}^{2}}$,

$\cos 2 \phi=\tilde{B}_{1} / \sqrt{\tilde{B}_{1}^{2}+\tilde{B}_{3}^{2}}, \quad \sin 2 \phi=-\tilde{B}_{3} / \sqrt{\tilde{B}_{1}^{2}+\tilde{B}_{3}^{2}}$,

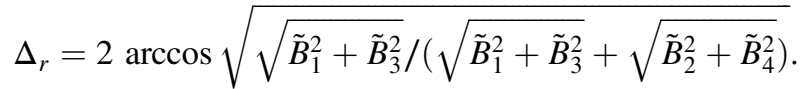

For lossless polarization systems, the angles $v$ and $\phi$ determine the orientation of the primary and secondary characteristic axes as defined in Refs. 60, 61. It follows from Eq. (5) that the transfer factor $t_{P A}\left(\vartheta, \vartheta^{\prime}\right)$ reaches its maximum value at $(\vartheta+$ $\left.\vartheta^{\prime}\right) / 2=v$ and $\vartheta^{\prime}-\vartheta=\phi$, i.e., the angles $v$ and $\phi$ describe the orientation of the polarizer and analyzer with which the domain 
being considered looks most bright [see Fig. 1(c)]. We will call the axis parallel to the sample plane and making the angle $v$ with the $x$-axis a polarization midaxis. According to Eq. (6), for any domain we can always find two mutually perpendicular polarization midaxes [if $v=v_{\mathrm{o}}$ satisfies Eq. (6), $v=v_{\mathrm{o}}+\pi / 2$ is also a solution of Eq. (6)]. Note that the angles $\phi$ and $\Delta_{r}$ of a domain are invariant with respect to any azimuthal rotations of this domain.

If the sample is a layer of a locally uniaxial medium, for homogeneous regions of the layer and inhomogeneous regions without azimuthal variations of the local optic axis across the layer thickness $\phi=0$; the polarization midaxes are oriented along the slow and fast axes of the region, and the characteristic angle $\Delta_{r}$ is related to the phase retardation $\delta$ in the region as follows:

$$
\Delta_{r}=2 \arccos (|\cos (\delta / 2)|) .
$$

This relation will also be valid for regions with small azimuthal variations of the local optic axis across the layer thickness in which light propagates in quasiadiabatic mode, ${ }^{62,63}$ with $\delta$ being the sum phase retardation in the region. In both these cases and for the mode of light propagation that is considered in Sec. 6, one of the polarization midaxes exactly (if $\phi=0$ ) or approximately indicates the direction of preferred orientation of the local slow axis over the entire thickness of the sample. We will call this axis the slow midaxis, and the second one the fast midaxis. In the case of biological tissues such as sclera and dermis, the orientation of the midaxes over the tissue area changes continuously everywhere or almost everywhere; therefore, for differentiation between the slow midaxis and the fast midaxis over the entire area being mapped it is sufficient to find experimentally which of the polarization midaxes is slow at one or several points of the mapping area. This can be done using a compensator in areas with small values of $\phi$ (see, e.g., Ref. 22). We used this method in the present study.

It should be noted that nonzero values of $\phi$ always point to the presence of structural chirality in the medium $(\phi=0$ for optical systems with a symmetry plane or the center of symmetry), and in many situations this characteristic angle can be used as a quantitative measure of the degree of manifestation of the structural chirality of the sample in its optical properties (in its observed optical chirality). In this study, we use the mapping of $\phi$ to estimate the degree and nature of the effect of azimuthal variations of the local optic axis across the sample thickness - the only factor that can lead to optical chirality in our situation —on the optical properties of the sample.

\section{Experimental Technique and Equipment}

In this study we employed a TPM mapping technique which uses the linear polarizer-sample-linear analyzer configuration. The sample is illuminated with light from a broadband source passed through a narrowband (interference) filter, which selects the required quasimonochromatic component. Images of the sample are acquired using a charge-coupled device (CCD) camera. From sample images obtained with different orientations of the polarizer and analyzer, maps of parameters characterizing the dependence of intensity in the image on the orientation of the polarizer and analyzer are calculated. Then, the characteristic angles $v, \phi$, and $\Delta_{r}$ are computed.

The measuring part of the experimental setup that we used was built on the basis of a polarization microscope POLAM L213 (LOMO, St. Petersburg, Russia). Images were captured with a 12-bit-grayscale CCD camera Videoscan-285/P-2001 (Videoscan, Moscow, Russia) mounted on the microscope. We use a specific image-acquisition technique ${ }^{64}$ which allows correction of the images for source intensity fluctuations and CCD dark current noise during data processing. Moreover, when processing the raw images, partial polarization of the light incident on the polarizer and image shifts that occur when the analyzer is rotated are taken into account. In the microscope, the degree of polarization of the light incident on the polarizer is close to 0.1 and slightly changes with wavelength. Taking this fact into account, in the data processing, the pixel intensities in the image being treated are multiplied by a calibration coefficient corresponding to the actual polarizer orientation and the spectral filter used. Areas to be mapped are always chosen somewhat smaller than original images. The image shift effect is eliminated by shifting borders of the area being cut from the original image. All measurements for this paper were made with a $2.5 \times$ objective, $\mathrm{NA}=0.05$.

We use the following expression for the image intensity $i_{I}\left(\vartheta, \vartheta^{\prime}\right)$ :

$$
\begin{aligned}
i_{I}= & B_{0}+B_{1} \cos \eta^{-}+B_{2} \cos \eta^{+}+B_{3} \sin \eta^{-}+B_{4} \sin \eta^{+} \\
& +B_{5} \cos 2 \vartheta+B_{6} \sin 2 \vartheta+B_{7} \cos 2 \vartheta^{\prime}+B_{8} \sin 2 \vartheta^{\prime}
\end{aligned}
$$

[cf., Eq. (3)]. In the case of quasimonochromatic illumination, the coefficients $B_{j}(j=0,1, \ldots 8)$ can be expressed as follows:

$B_{j}=\tilde{B}_{j}(\tilde{\lambda}) K I_{0}$

where $I_{0}$ is the intensity of the light incident on the sample, $K$ is an instrumental coefficient, and $\tilde{\lambda}$ is the mean wavelength of the incident light. Determining the coefficients $B_{j}$ is the primary task in the technique we use. In this work, the maps of $B_{j}$ were calculated by the least-square method from 16 images of the sample obtained with $\vartheta=-45 \mathrm{deg}, 0 \mathrm{deg}, 45 \mathrm{deg}, 90 \mathrm{deg}$ and $\vartheta^{\prime}=-45 \mathrm{deg}, 0 \mathrm{deg}, 45 \mathrm{deg}, 90 \mathrm{deg}$. The angles $v, \phi$, and $\Delta_{r}$ were computed using Eqs. (6)-(8) with $\tilde{B}_{j}$ replaced by $B_{j}$.

To estimate the applicability of Eq. (10) and the accuracy of mapping results, we used the following parameter:

$\varepsilon_{\mathrm{te}}=\frac{\left\langle\sqrt{\frac{1}{16} \sum_{k=1}^{16}\left(i_{k \mathrm{e}}-i_{k \mathrm{e}}\right)^{2}}\right\rangle}{2\left\langle B_{0}\right\rangle}$,

where the angle brackets denote averaging over the mapped area, $i_{\text {kte }}$ is the intensity of a pixel in the $k$ th image computed using the obtained $B_{j}$ values, and $i_{\mathrm{ke}}$ is the intensity of the same pixel in the corresponding corrected experimental image.

\section{Samples}

In our experiments, we used samples of rat sclera, porcine sclera, and rat skin, all being optically cleared with glycerol or a glycerol solution. Also, for illustrative purposes, we used a sample of rat tail tendon. The studies were approved by the Ethics Committee of Saratov State Medical University (Saratov, Russia).

Before optical clearing, the sclera samples were mechanically cleared of the choroid and retina. The samples of porcine sclera were also cleared of episcleral tissue from the outside. The skin samples (from back and abdomen regions) were 
mechanically cleared of adipose tissue and hair. After that processing, the samples were placed in pure glycerol (analytical grade, $99.3 \mathrm{wt} \%$ purity) or $50 \mathrm{vol} \%$ aqueous solution of this reagent. Sclera samples were optically cleared in pure glycerol. Pure glycerol or $50 \mathrm{vol} \%$ glycerol solution was used for clearing of skin samples. The samples of porcine sclera and rat skin were incubated in glycerol for two days, and the samples of rat sclera for several hours. After clearing, the samples of sclera looked clear and colorless, the skin samples cloudy and slightly colored. When examined under the polarizing microscope with crossed polarizers and achromatic illumination, the sclera and skin samples did not show very bright areas and interference colors, which indicated that the phase retardation caused by birefringence is much smaller than $180 \mathrm{deg}$ over the entire area of the sample. This was confirmed by evaluation of the retardance of these samples using a Babinet compensator. The microscopic measurements were performed with the samples placed in pure glycerol $/ 50$ vol\% glycerol solution between an object-plate and a cover glass.

\section{Experimental Results and Discussion}

In Figs. 2 and 3, we show typical mapping results for samples of porcine sclera, rat sclera, and rat skin. Figure 2 shows the maps of the slow midaxis orientation, $\phi$, and $\Delta_{r}$ for the porcine sclera cleared with pure glycerol (Sample 1) and the obtained estimates for an effective birefringence index of the medium, which was calculated as

$$
\Delta n_{\mathrm{eff}}=\frac{\Delta_{r} \lambda}{2 \pi d},
$$

where $d$ is the thickness of the sample, for various regions of the sample. Wavelength dependences of the area-averaged value of $\Delta n_{\text {eff }}$, denoted as $\left\langle\Delta n_{\text {eff }}\right\rangle$, for three small regions highlighted by squares on the maps and for the entire area being mapped are shown. Figure 3 shows the maps of $\phi$ for different wavelengths and the corresponding distribution functions of $\phi$ for Sample 1, the sample of rat sclera cleared with pure glycerol (Sample 2), and two samples of rat skin of which one is cleared in $50 \mathrm{vol} \%$ glycerol solution and the other in pure glycerol (Sample 3 and Sample 4, respectively). The mean and the maximum values of $\Delta_{r}$ over the area being mapped are also shown. In all the cases, the value of $\varepsilon_{t \mathrm{e}}$ did not exceed $1.5 \times 10^{-2}$; in most cases it was less than $1 \times 10^{-2}$. The collimated transmittance for the porcine sclera sample was $19 \%$ to $40 \%$, for the rat sclera sample about $80 \%$, for the rat skin sample cleared with $50 \mathrm{vol} \%$ glycerol $7 \%$ to $14 \%$, and for the rat skin cleared with pure glycerol $4 \%$ to $9 \%$.

Let us start our discussion with a consideration of a 1-D analog of the optical problem at hand. Consider a system of $N$ phase plates with a sum phase retardation $G=\delta_{1}+\delta_{2}+\ldots$ $+\delta_{N}$, where $\delta_{j}=h_{j} G$ is the phase retardation of the $j$ th plate $\left(h_{1}+h_{2}+\ldots+h_{N}=1\right)$. Let a light wave fall on this system in the normal direction, along the $z$-axis of a reference system $(x, y, z)$, successively passing through plates with numbers 1,2 ,

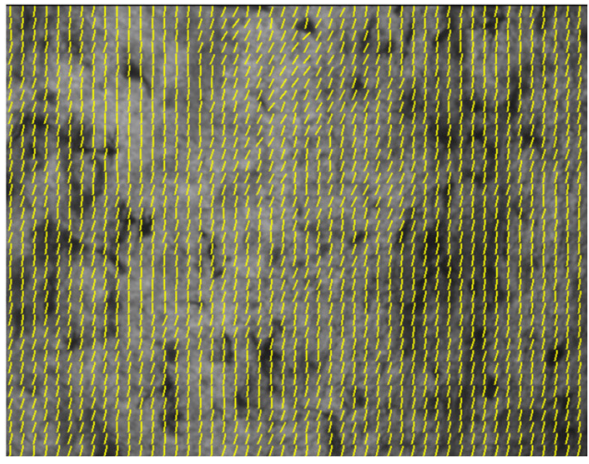

(a)

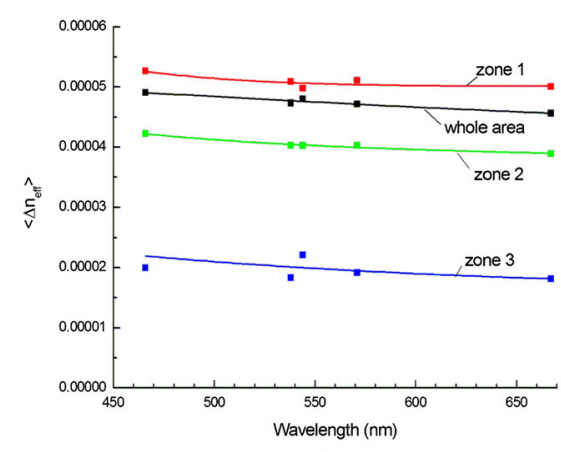

(c)

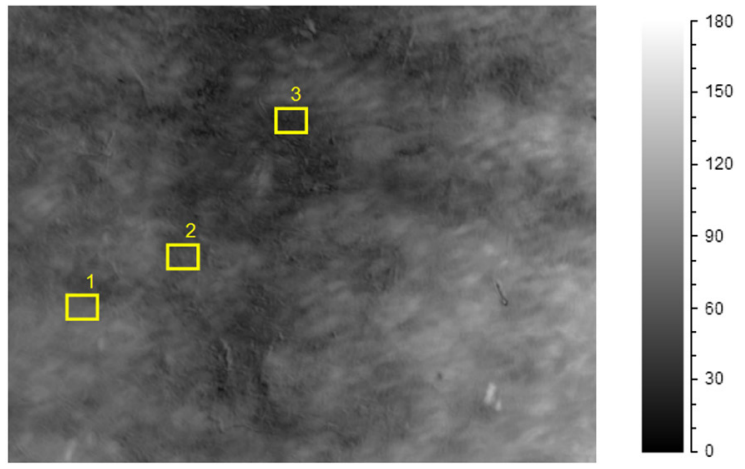

(b)

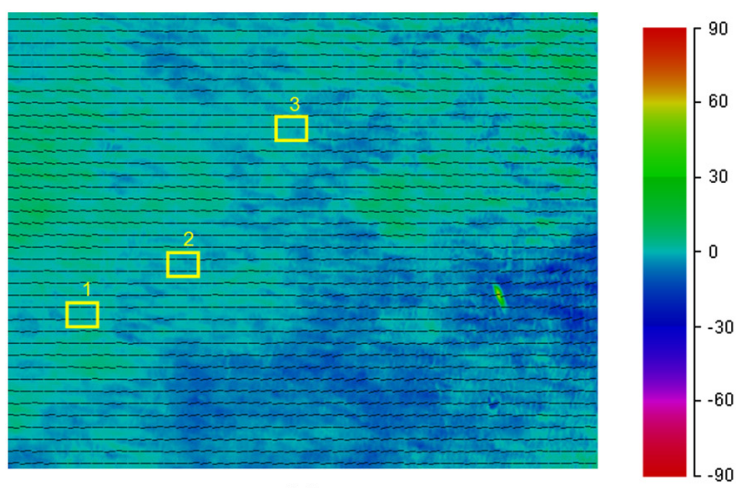

(d)

Fig. 2 Mapping results for a 1.75-mm-thick porcine sclera sample (Sample 1 ) at $\lambda=544 \mathrm{~nm}$. (a) Map of the slow midaxis orientation superimposed on the unpolarized image of the sample. (b) Map of $\Delta_{r}$. (c) Wavelength dependences of average values of $\Delta n_{\text {eff }}$ for the whole area and for zones 1-3 highlighted by rectangles in (b) and (d). Boxes show experimental values. (d) Map of $\phi$. The mapped area width is $1.54 \mathrm{~mm}$. 

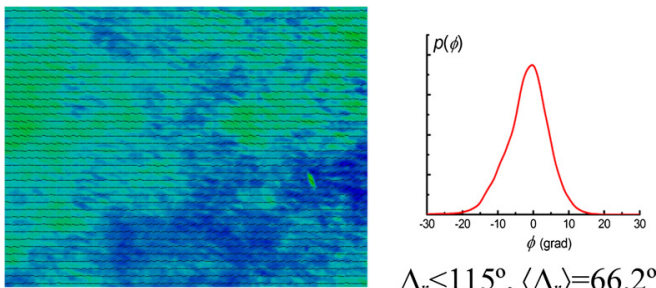

$\Delta_{r}<115^{\circ},\left\langle\Delta_{r}\right\rangle=66.2^{\circ}$

$\lambda=466 \mathrm{~nm}$
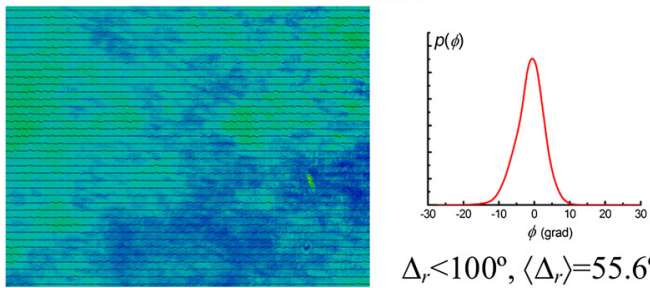

$\Delta_{r}<100^{\circ},\left\langle\Delta_{r}\right\rangle=55.6^{\circ}$

$\lambda=544 \mathrm{~nm}$
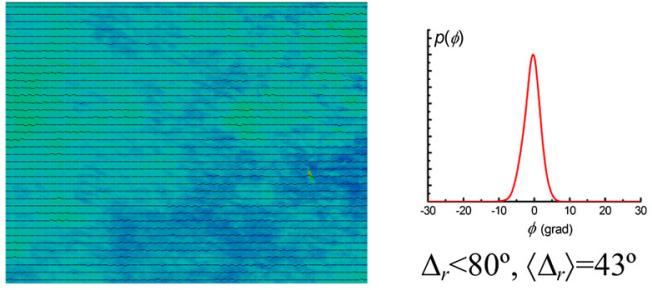

$\Delta_{r}<80^{\circ},\left\langle\Delta_{r}\right\rangle=43^{\circ}$

$\lambda=667 \mathrm{~nm}$

(a)
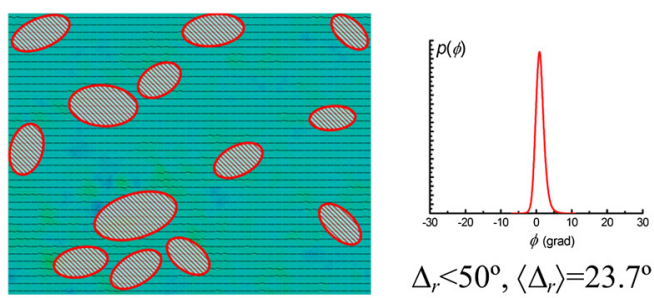

$\Delta_{r}<50^{\circ},\left\langle\Delta_{r}\right\rangle=23.7^{\circ}$

$\lambda=466 \mathrm{~nm}$
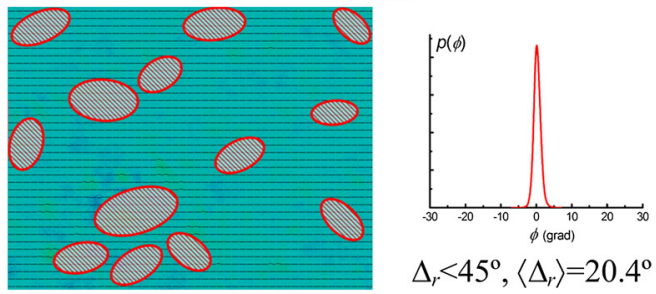

$\Delta_{r}<45^{\circ},\left\langle\Delta_{r}\right\rangle=20.4^{\circ}$

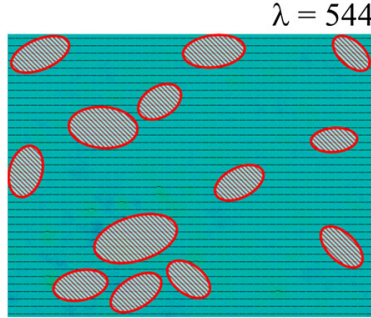

$\lambda=667 \mathrm{~nm}$

(c)

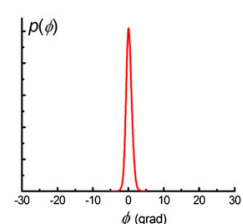

$\Delta_{r}<35^{\circ},\left\langle\Delta_{r}\right\rangle=16.8^{\circ}$
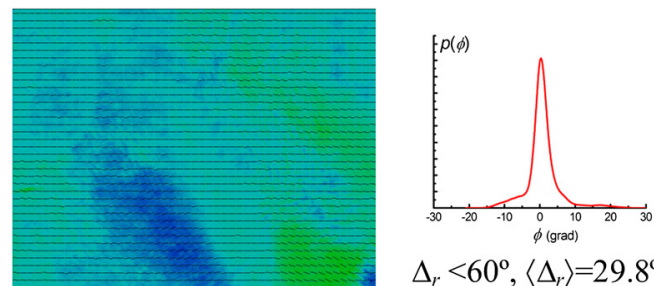

$\Delta_{r}<60^{\circ},\left\langle\Delta_{r}\right\rangle=29.8^{\circ}$

$\lambda=466 \mathrm{~nm}$
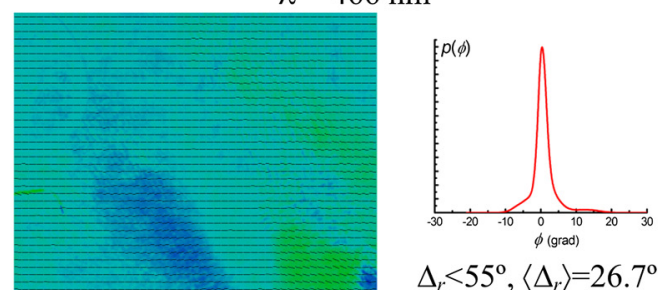

$\Delta_{r}<55^{\circ},\left\langle\Delta_{r}\right\rangle=26.7^{\circ}$ $\lambda=544 \mathrm{~nm}$
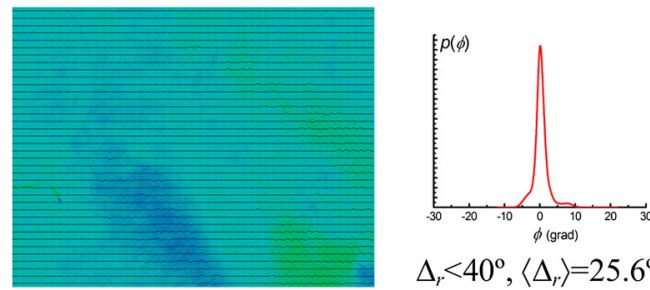

$\Delta_{r}<40^{\circ},\left\langle\Delta_{r}\right\rangle=25.6^{\circ}$

$\lambda=667 \mathrm{~nm}$

(b)
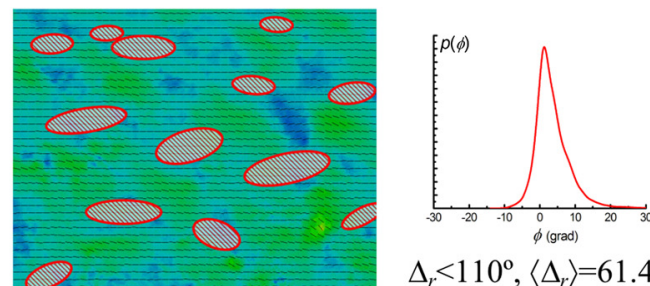

$\Delta_{r}<110^{\circ},\left\langle\Delta_{r}\right\rangle=61.4^{\circ}$
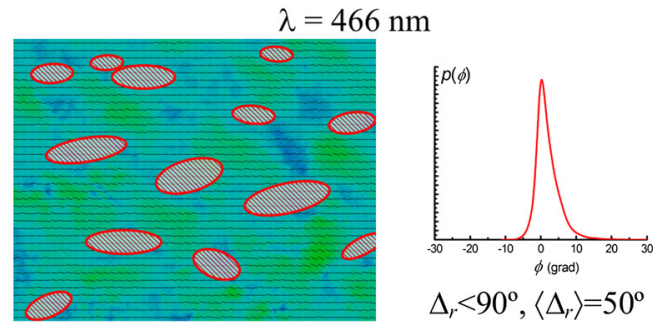

$\Delta_{r}<90^{\circ},\left\langle\Delta_{r}\right\rangle=50^{\circ}$

$\lambda=544 \mathrm{~nm}$
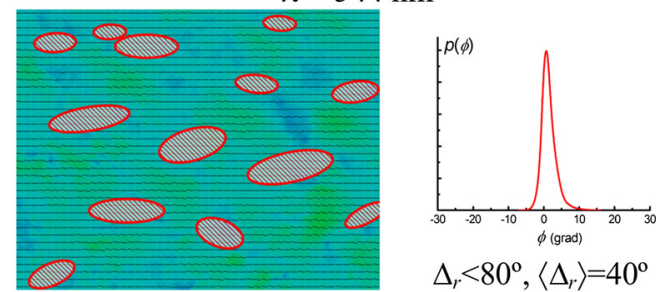

$\Delta_{r}<80^{\circ},\left\langle\Delta_{r}\right\rangle=40^{\circ}$

$\lambda=667 \mathrm{~nm}$

(d)

Fig. 3 Maps of characteristic angle $\phi$ and corresponding distribution functions of $\phi$ at different wavelengths for (a) Sample 1 (porcine sclera), the thickness of the sample is $1.75 \mathrm{~mm}$; (b) Sample 2 (rat sclera), $190 \mu \mathrm{m}$; (c) Sample 3 (rat skin), $1.07 \mathrm{~mm}$; and (d) Sample 4 (rat skin), $1.64 \mathrm{~mm}$. In (c) and (d), the hatched ellipses mark areas occupied by hair follicles. The mapped area width is $1.54 \mathrm{~mm}$. 
and so on. Using the unitary unimodular representation of Jones matrices, we can express the Jones matrix of this system, referred to the basis $(x, y)$, as follows

$$
\mathbf{P}=\mathbf{P}_{N} \mathbf{P}_{N-1} \cdot \ldots \cdot \mathbf{P}_{2} \mathbf{P}_{1}
$$

where

$\mathbf{P}_{j}=\left[\begin{array}{cc}\cos \left(G h_{j} / 2\right)+\mathrm{i} \sin \left(G h_{j} / 2\right) \cos 2 \varphi_{j} & \mathrm{i} \sin \left(G h_{j} / 2\right) \sin 2 \varphi_{j} \\ \mathrm{i} \sin \left(G h_{j} / 2\right) \sin 2 \varphi_{j} & \cos \left(G h_{j} / 2\right)-\mathrm{i} \sin \left(G h_{j} / 2\right) \cos 2 \varphi_{j}\end{array}\right]$

Here $\varphi_{j}$ is the angle between the slow axis of the $j$ th phase plate and the $x$-axis.

Suppose that $G$ is so small that $\cos (G / 2) \approx 1$. Then, we can use the approximations $\cos \left(G h_{j} / 2\right) \approx 1$ and $\sin \left(G h_{j} / 2\right) \approx$ $G h_{j} / 2$ to represent the matrix $\mathbf{P}_{j}$ as

$\mathbf{P}_{j} \approx \mathbf{U}+\mathrm{i} \frac{G h_{j}}{2}\left(\begin{array}{cc}\cos 2 \varphi_{j} & \sin 2 \varphi_{j} \\ \sin 2 \varphi_{j} & -\cos 2 \varphi_{j}\end{array}\right)$,

where $\mathbf{U}$ is a unit matrix. On substituting Eq. (16) into Eq. (14), we have

$\mathbf{P} \approx \mathbf{U}+\mathrm{i} \frac{G}{2}\left(\begin{array}{cc}\sum_{j=1}^{N} h_{j} \cos 2 \varphi_{j} & \sum_{j=1}^{N} h_{j} \sin 2 \varphi_{j} \\ \sum_{j=1}^{N} h_{j} \sin 2 \varphi_{j} & -\sum_{j=1}^{N} h_{j} \cos 2 \varphi_{j}\end{array}\right)$

This relation can be rewritten as

$$
\mathbf{P} \approx \mathbf{U}+\mathrm{i} \frac{G S}{2}\left(\begin{array}{cc}
\cos 2 \chi & \sin 2 \chi \\
\sin 2 \chi & -\cos 2 \chi
\end{array}\right)
$$

where

$$
\begin{array}{r}
S=\sqrt{\bar{X}^{2}+\bar{Y}^{2}}, \quad \cos 2 \chi=\frac{\bar{X}}{S}, \quad \sin 2 \chi=\frac{\bar{Y}}{S}, \\
\bar{X}=\sum_{j=1}^{N} h_{j} \cos 2 \varphi_{j}, \quad \bar{Y}=\sum_{j=1}^{N} h_{j} \sin 2 \varphi_{j} .
\end{array}
$$

Comparing Eq. (17) with Eq. (16), we see that in the case under consideration $[\cos (G / 2) \approx 1]$ the system behaves as an ordinary (equivalent) phase plate whose phase retardation is equal to $G S$, and whose slow axis is oriented at angle $\chi$ with respect to the $x$-axis. Note that here $S$ and $\chi$ are invariant with respect to any permutations of phase plates. Let the plates have the same birefringence index $\Delta n_{\mathrm{p}}$ and a sum thickness $d_{\mathrm{p}}\left(G=2 \pi \Delta n_{\mathrm{p}} d_{\mathrm{p}} / \lambda\right)$ and be separated by isotropic layers, $d$ being the thickness of the whole system. Then, the birefringence index $\Delta n_{\mathrm{ep}}$ of the equivalent plate of thickness $d$ can be expressed as

$\Delta n_{\mathrm{ep}}=\Delta n_{\mathrm{p}} C_{\mathrm{p}} S$

where $C_{\mathrm{p}}=d_{\mathrm{p}} / d$ is "the volume fraction" of phase plates in the system. The parameter $S$ in this case is a purely geometrical factor and can be considered as a measure of the orientational order of phase plates. The direction that is specified by the angle $\chi$ can be regarded as the preferred direction of orientation of phase plates in the system. At the microlevel, the analogs of phase plates of the system and $\Delta n_{\mathrm{ep}}$ are collagen fibers and the effective birefringence index of the tissue. With $\Delta n_{\mathrm{p}}=0.0015$ (a rough estimate for collagen fibers in a tissue cleared with glycerol) and $C_{\mathrm{p}}=0.2\left(\operatorname{skin}^{53}\right)$, a reliable estimate for the $d$ value up to which the system will behave as a single phase plate in the visible region is $40 \mu \mathrm{m}$. Based on this, we may take $40 \mu \mathrm{m}$ as an optimum linear size of the elementary volume with which the macroscopic optic axis and the macroscopic $\Delta n$ of the cleared tissue are associated.

When dealing with a thick sample of the tissue $(>100 \mu \mathrm{m})$, we may regard it as a pile of slices with thicknesses of the order of $40 \mu \mathrm{m}$, supposing that within each slice $\Delta n$ and $\mathbf{c}$ depend on $x$ and $y$ but are independent of $z$. The preceding analysis is often applicable to thick samples of glycerol-cleared derma and sclera because the maximum values of the macroscopic birefringence index $\Delta n$ for these tissues are significantly smaller than the value of $\Delta n_{\mathrm{p}} C_{\mathrm{p}}$ in the previous example. To obtain some useful estimates for this case, we slightly refine Eq. (17) as:

$$
\begin{aligned}
\mathbf{P} & =\left(\begin{array}{cc}
a^{\prime}+\mathrm{i} a^{\prime \prime} & b^{\prime}+\mathrm{i} b^{\prime \prime} \\
-b^{\prime}+\mathrm{i} b^{\prime \prime} & a^{\prime}-\mathrm{i} a^{\prime \prime}
\end{array}\right) \\
& =\mathbf{U}+\mathrm{i} \frac{G S}{2}\left(\begin{array}{cc}
\cos 2 \chi & \sin 2 \chi \\
\sin 2 \chi & -\cos 2 \chi
\end{array}\right)+O\left(G^{2}\right)
\end{aligned}
$$

as $G \rightarrow 0$. In this case, the phase plates of the system are analogs of slices in the macromodel. The characteristic angle $\phi$ of this system can be expressed in terms of elements of $\mathbf{P}$ as

$\phi=\arctan \left(-b^{\prime} / a^{\prime}\right)$.

From Eq. (19) we see that $b^{\prime}=O\left(G^{2}\right)$ and $a^{\prime}=1+O\left(G^{2}\right)$ as $G \rightarrow 0$, and from Eq. (20) we have $\phi=O\left(G^{2}\right)$, i.e., when $G$ approaches zero, $\phi$ decreases proportionally to $G^{2}$. This means that, on the one hand, there is a relatively wide range of $G$ values for which the values of $\phi$ are very small ( $\cos G \approx 1$ ). We will call this range of $G$ the range of negligible optical chirality (for this range, the structural chirality of the system practically does not manifest itself in optical properties of the system) and denote the value of $G$ on the boundary of this range as $G_{\mathrm{NOC}}$. On the other hand, outside this range $\phi$ increases rapidly with $G$. Figure 4 illustrates this. Figure 4(a) shows the dependence of $\phi$ on $G$ for 20 randomly generated four-layer structures. Based on what we see in this figure, we can take $G_{\mathrm{NOC}}=20 \mathrm{deg}$. Figures 4(b) and 4(c) show how accurately the preferred direction of orientation of the plates and the phase retardation $G S$ for these systems can be determined using the estimates $v \approx \chi$ (here and in what follows we assume that the angle $v$ specifies the orientation of the slow midaxis) and $\Delta_{r} \approx G S$. We see that when $G<G_{\mathrm{NOC}}, \chi$ and $G S$ can be determined very accurately. We also see that outside the range of negligible optical chirality the estimate $v \approx \chi$ is also good in a large range of $G$ values. The accuracy of the estimate $\Delta_{r} \approx G S$ when $G>G_{\mathrm{NOC}}$ is on the whole worse than the accuracy of the estimate $v \approx \chi$, but can also be considered acceptable up to $G=40 \mathrm{deg}$. Note that for configurations with $\phi=0$, $\Delta_{r}=G S$ for any $G$. If $\phi$ is small, the estimate $\Delta_{r} \approx G S$ can provide acceptable accuracy at large values of $G$ as well. In 

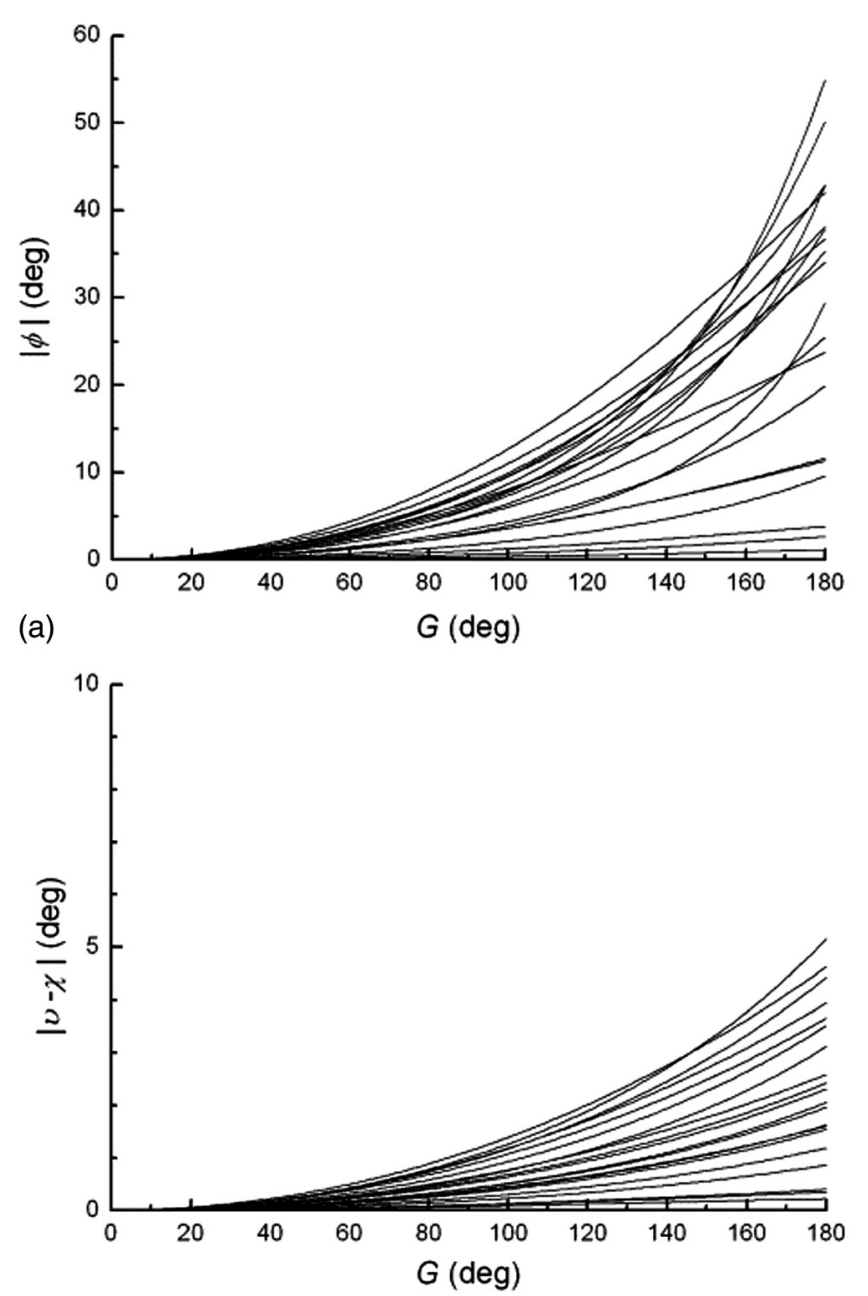

(b)

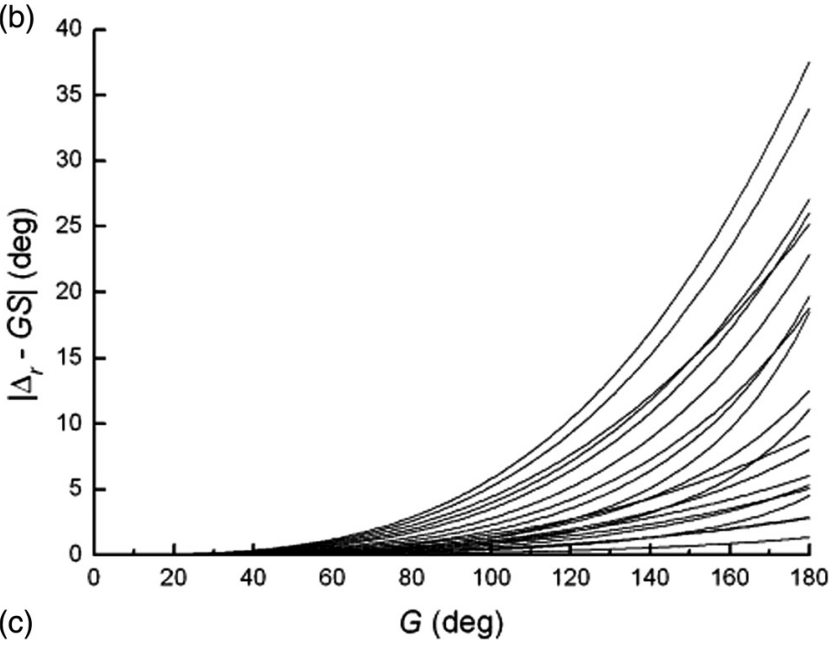

Fig. 4 Computer modeling results for 20 randomly generated fourlayer structures consisting of phase plates. Dependence of characteristic angle $\phi(a)$, the accuracy of estimation of the direction of preferred orientation of phase plates (b), and effective phase retardation GS (c) on the sum phase retardation $G$.

Fig. 5, we present the estimates for maximum deviation, $\max \left|\Delta_{r}-G S\right|$, for $|\phi|<2 \mathrm{deg},|\phi|<5 \mathrm{deg}$, and $|\phi|<10 \mathrm{deg}$. These estimates were obtained on an ensemble of 50,000 randomly generated four-layer systems.

It can be seen from the values of $\Delta_{r}$ shown in Fig. 3 that the condition of negligible optical chirality in the visible range is not

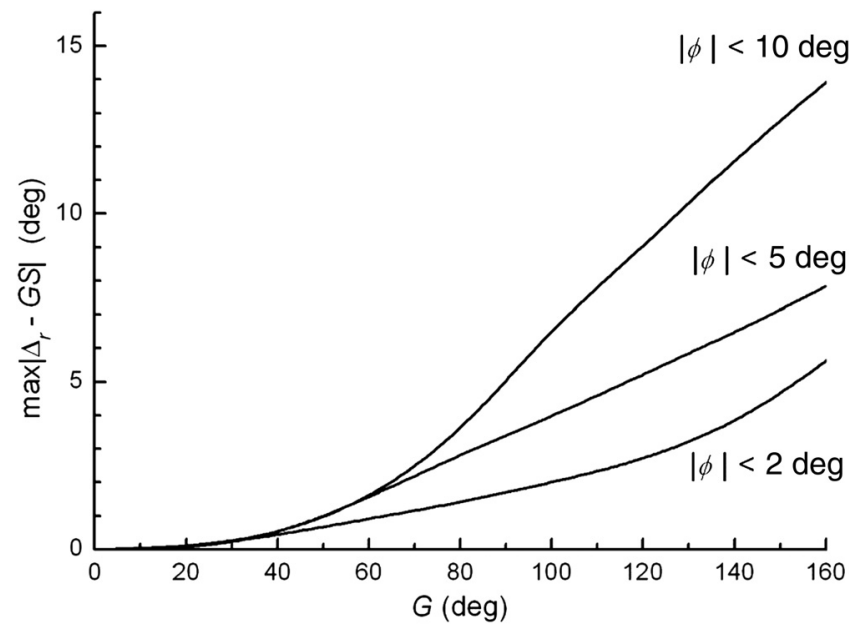

Fig. 5 Dependence of the maximum value of $\left|\Delta_{r}-G S\right|$ on the sum phase retardation $G$ for an ensemble of 50,000 randomly generated four-layer structures under different restrictions on $\phi$.

satisfied for the sclera and derma samples under consideration, and on all the maps we see some variation of $\phi$. For all samples, the average value of $\phi$ is approximately zero, and $\phi$ varies within a relatively narrow interval. It can be seen that with increasing wavelength the values of $\phi$ in areas where $\phi$ differs appreciably from zero decrease in magnitude, i.e., the variance of $\phi$ decreases with increasing $\lambda$ (this is clearly seen from the plots of the distribution function of $\phi$ in Fig. 3). This fact is in complete agreement with what we see in Fig. 4(a): an increase in $\lambda$ leads to reduction of $G$ and, consequently, to the reduction of $|\phi|$.

For any of the maps, the maximum value of $|\phi|$ will not exceed those predicted by the 1-D model if we take $G$ equal to the maximum $\Delta_{r}$ on this map, or a little higher. The above estimates show that for the values of $\Delta_{r}$ and $\phi$ that we have for our samples the accuracy of the relations $v \approx \chi$ and $\Delta_{r} \approx G S$ is good over the whole visible region or at least in the red end of the spectrum. This gives us a reason to believe that the slow midaxis is oriented in the direction of preferred orientation of collagen fibers, averaged over the sample thickness, not only in the areas with $\phi \approx 0$, but throughout the area of mapping, and adopt the effective birefringence index given by Eq. (13) as a quantity characterizing the degree of orientational order and the packing density of collagen fibers with averaging over the sample thickness.

As an example of a situation in which such assessment is impossible, in Fig. 6 we show the results of mapping for the sample of rat tail tendon. In this case, we deal with a $1-\mathrm{mm}$ thick sample of rat tail tendon (tertiary tendon bundle) cleared with glycerol. The tendons are characterized by high packing density and high short-range orientational order of collagen fibers. Therefore, the tendon has significantly higher values of $\Delta n$ than the sclera and dermis. Thus, for this sample, viewed between crossed polarizers, interference colors were observed $(\delta>180 \mathrm{deg})$. At the same time, the local average direction of orientation of collagen fibers in the tissue varies significantly over distances of the order of hundreds of microns. In our case, this is reflected in the rapid and strong variations of the angles $v$ and $\phi$ (Fig. 6).

Figures 7 and 8 show results of mapping for a rat skin sample (Sample 4) and a rat sclera sample (Sample 2). We give these maps to demonstrate some features of the organization of 


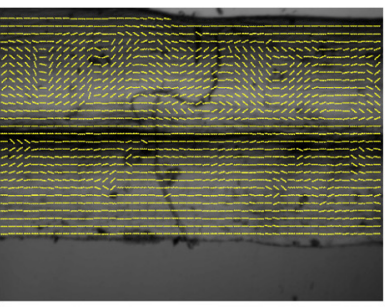

(a)

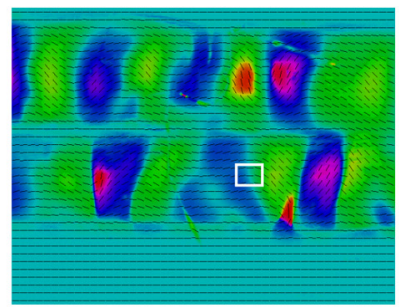

(b)

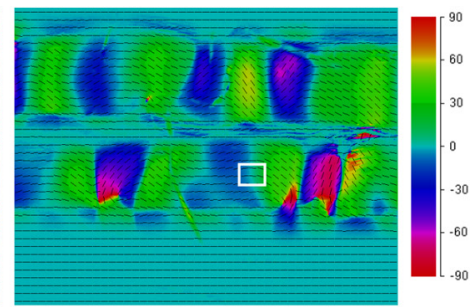

(c)

Fig. 6 Mapping results for a 1-mm-thick sample of rat tail tendon. (a) Map of the slow midaxis orientation superimposed on the unpolarized image of the sample. Parts (b) and (c) are maps of $\phi$ at $\lambda=466 \mathrm{~nm}$ and $\lambda=667 \mathrm{~nm}$, respectively. The mapped area width is $1.54 \mathrm{~mm}$.

collagen tissue structure that can be revealed through the polarization mapping.

Figure 7 shows the results for the rat skin sample. There are several clusters of hair follicles in the area being mapped. As can be seen from the map of orientation of the slow midaxis [Fig. 7(a)], the lines of preferred orientation of collagen fibers tend to flow around the hair clusters. At the same time, from the map of $\phi$-in particular, from how $\phi$ changes when we pass around a hair cluster (for instance, we may see clusters for which $\phi$ changes in sign four times when we make a turn around the cluster) - we may conclude that under the dermis layer in which the flow-around occurs there is a deep layer with substantially slower variation of the direction of preferred orientation of collagen fibers. This pattern might be expected since the hair follicles penetrate deep into the dermis by about two-thirds of the dermis thickness.

Figure 8 shows the results of mapping of a rat sclera region containing a blood vessel. From these maps, it is clear that the collagen structure organization in the vicinity of the vessel is connected with its presence. It can be seen that the vessel goes within a strip with relatively low values of $\Delta_{r}$. Outside this strip, $\Delta_{r}$ on the average is considerably higher. Thus, it may be concluded that in the vicinity of the vessel the collagen structure is more amorphous.

Note, finally, one more utility of the TPM mapping technique that can be used when dealing with tissue samples having a large

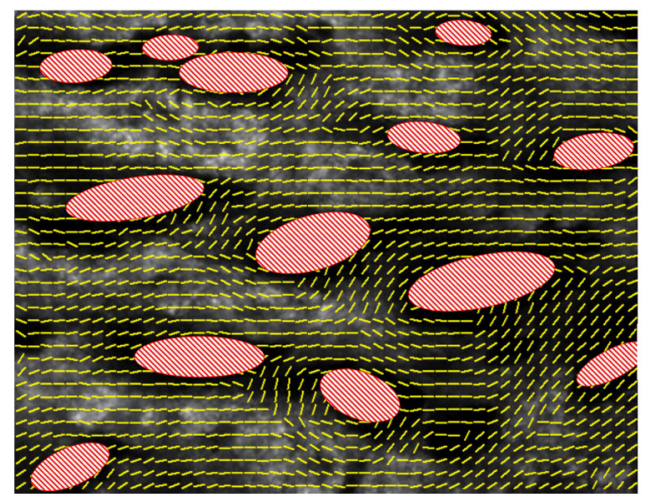

(a)

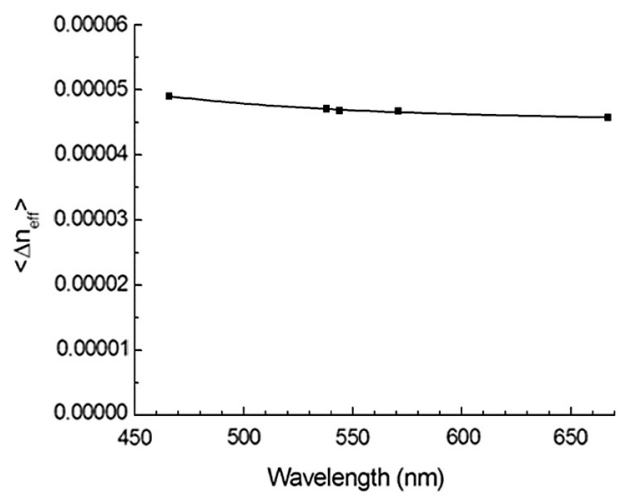

(c)

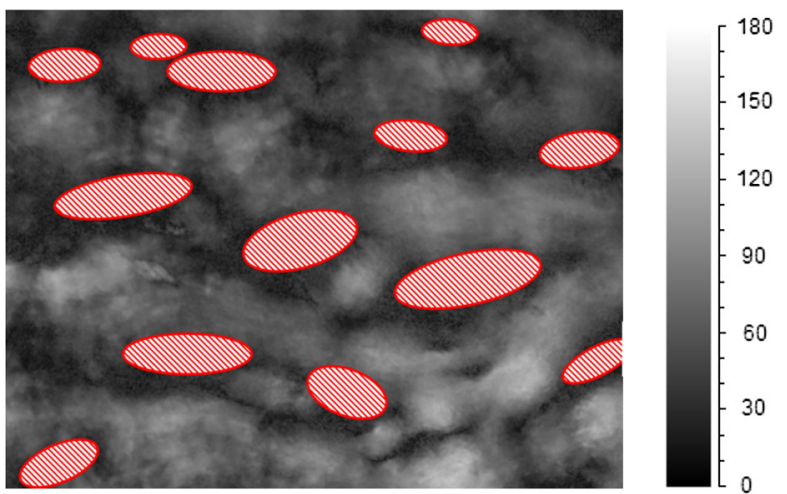

(b)

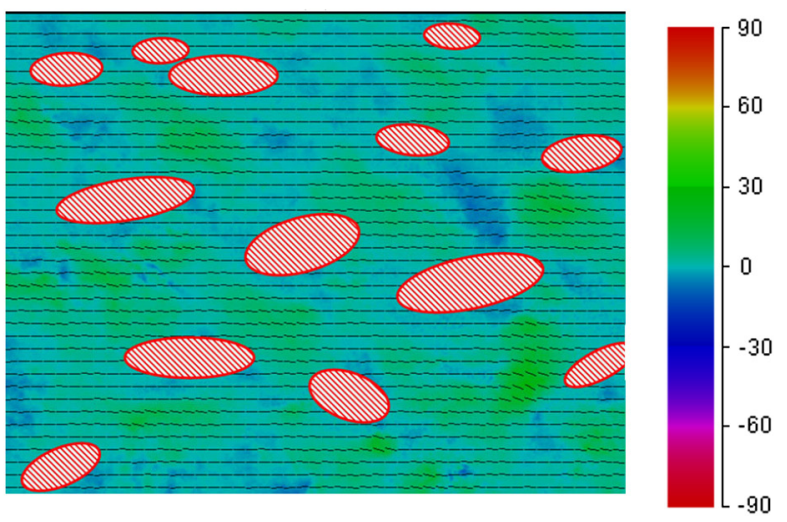

(d)

Fig. 7 Mapping results for a 1.64-mm-thick rat skin sample (Sample 4) at $\lambda=544 \mathrm{~nm}$. (a) Map of the slow midaxis orientation superimposed on the unpolarized image of the sample. (b) Map of $\Delta_{r}$. (c) Wavelength dependence of $\Delta n_{\text {eff }}$ averaged over the whole area. Boxes show experimental values. (d) Map of $\phi$. Hatched ellipses mark areas occupied by hair follicles in (a), (b), and (d). The mapped area width is $1.54 \mathrm{~mm}$. 


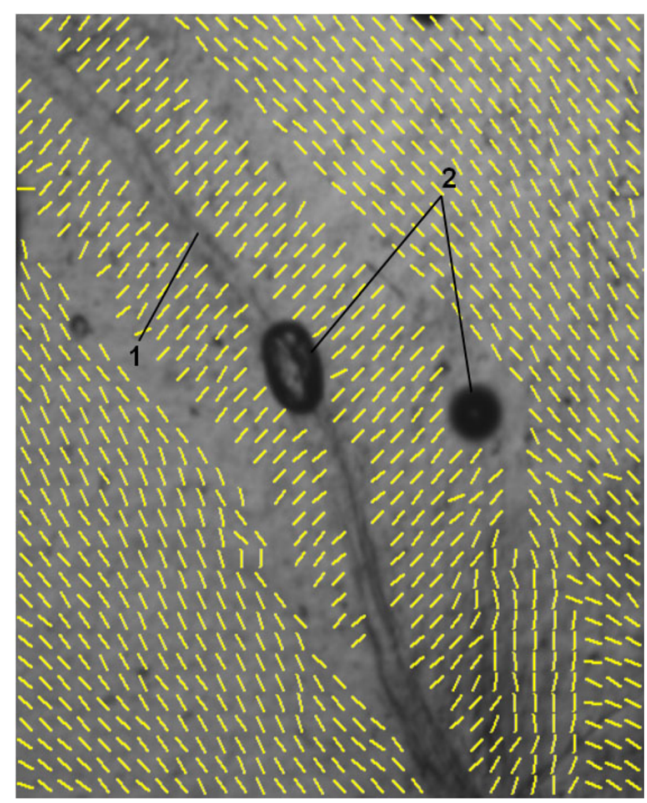

(a)

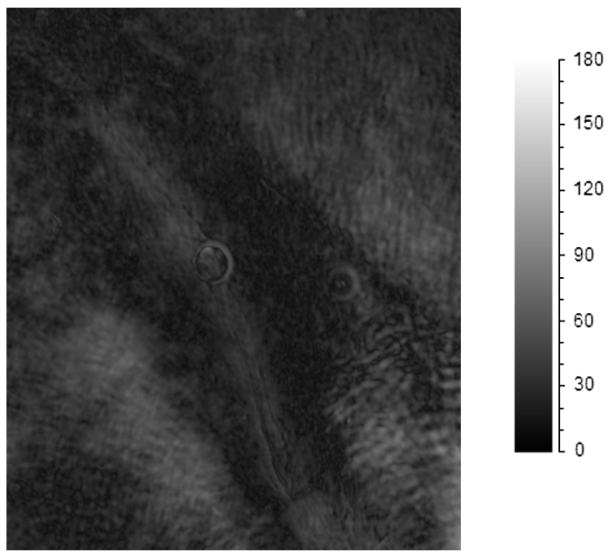

(b)

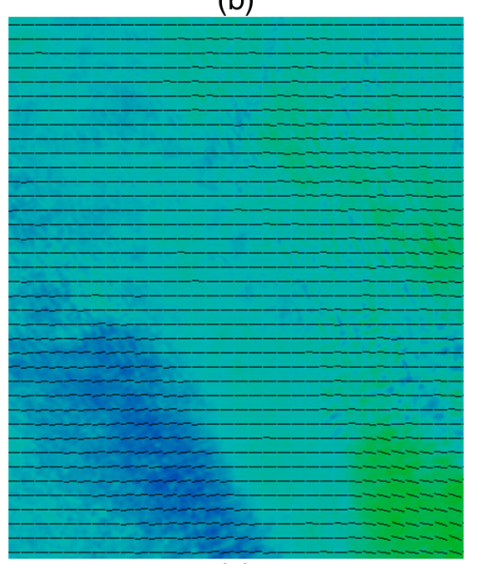

(c)

Fig. 8 Mapping results for a 190- $\mu$ m-thick rat sclera sample (Sample 2) at $\lambda=544 \mathrm{~nm}$. (a) Map of the slow midaxis orientation superimposed on the unpolarized image of the sample. 1-a blood-vessel, 2-air bubbles in glycerol. (b) Map of $\Delta_{r}$. (c) Map of $\phi$. The mapped area width is $1 \mathrm{~mm}$.

retardation, to which the discussed approximations cannot be applied. Conventional tools for measuring linear retardance are designed to work with samples for which $\phi=0$. The mapping allows one to find areas where $\phi$ is close to zero and gives for those areas the directions of the polarization midaxes, oriented approximately along the local slow and fast axes. One such area for the sample of tendon is highlighted by a rectangle in Fig. 6.

\section{Conclusions}

We have shown that in many cases low-NA polarization mapping allows accurate quantitative characterization of collagen fiber alignment in thick samples of sclera and dermis. Conditions under which the mapping can give accurate estimates for parameters of collagen fiber alignment in thick samples have been indicated. Simple theoretical models helpful in analysis of polarization mapping results for thick samples of optically cleared tissues have been presented. Also, we have given experimental estimates of the effective birefringence index as a function of wavelength for glycerol-cleared sclera and dermis.

\section{Acknowledgments}

This work had been performed in the framework of Research and Development RF government contract № 2014/203, Research № 1490.

\section{References}

1. V. V. Tuchin, Tissue Optics: Light Scattering Methods and Instruments for Medical Diagnosis, SPIE press, Bellingham, Washington (2007).

2. M. Wolman, "Polarized light microscopy as a tool of diagnostic pathology," J. Histochem. Cytochem. 23(1), 21-50 (1975).

3. S. Bancelin et al., "Determination of collagen fiber orientation in histological slides using Mueller microscopy and validation by second harmonic generation imaging," Opt. Express 22(19), 22561-22574 (2014).

4. M. Kim, D. Keller, and C. Bustamante, "Differential polarization imaging. I. Theory," Biophys. J. 52(6), 911-927 (1987).

5. D. A. Beach et al., "Differential polarization imaging. III. Theory confirmation. Patterns of polymerization of hemoglobin $\mathrm{S}$ in red blood sickle cells," Biophys. J. 53(3), 449-456 (1988).

6. M. Mujat, R. D. Ferguson, and N. Iftimia, "Mueller matrix microscopy," Proc. SPIE 8873, 88730E (2013).

7. R. Oldenbourg and G. Mei, "New polarized light microscope with precision universal compensator," J. Microsc. 180(2), 140-147 (1995).

8. M. Shribak and R. Oldenbourg, "Techniques for fast and sensitive measurements of two-dimensional birefringence distributions," Appl. Opt. 42(16), 3009-3017 (2003).

9. C. C. Hoyt and R. Oldenbourg, "Structural analysis with quantitative birefringence imaging," Am. Lab. 31(14), 34-42 (1999).

10. R. Oldenbourg, E. D. Salmon, and P. T. Tran, "Birefringence of single and bundled microtubules," Biophys. J. 74(1), 645-654 (1998).

11. P. T. Tran et al., "Muscle fine structure and microtubule birefringence measured with a new Pol-Scope," Biol. Bull. 187(2), 244-245 (1994).

12. D. Keefe et al., "Polarized light microscopy and digital image processing identify a multilaminar structure of the hamster zona pellucida," Hum. Reprod. 12(6), 1250-1252 (1997). 
13. L. Liu et al., "Increased birefringence in the meiotic spindle provides a new marker for the onset of activation in living oocytes," Biol. Reprod. 63(1), 251-258 (2000).

14. S. Inoué, "A method for measuring small retardations of structures in living cells," Exp. Cell. Res. 2(3), 513-517 (1951).

15. S. Inoue, "Polarization optical studies of the mitotic spindle. I. The demonstration of spindle fibers in living cells," Chromosoma $\mathbf{5}(1), 487-500$ (1953).

16. J. R. Kuhn, Z. Wu, and M. Poenie, "Modulated polarization microscopy: a promising new approach to visualizing cytoskeletal dynamics in living cells," Biophys. J. 80(2), 972-985 (2001).

17. T. T. Tower and R. T. Tranquillo, "Alignment maps of tissues: I. Microscopic elliptical polarimetry," Biophys. J. 81(5), 2954-2963 (2001).

18. T. T. Tower and R. T. Tranquillo, "Alignment maps of tissues: II. Fast harmonic analysis for imaging," Biophys. J. 81(5), 2964-2971 (2001).

19. A. G. Matoltsy and G. F. Odland, "Investigation of the structure of the cornified epithelium of the human skin," J. Biophys. Biochem. Cytol. 1(3), 191-196 (1955).

20. E. J. Naylor, "The structure of the cornea as revealed by polarized light," Q. J. Microsc. Sci. 3(25), 83-88 (1953).

21. L.-W. Jin et al., "Imaging linear birefringence and dichroism in cerebral amyloid pathologies," PNAS 100(26), 15294-15298 (2003).

22. P. Bullough and J. Goodfellow, "The significance of the fine structure of articular cartilage," J. Bone Joint Surg. Br. 50(4), 852-857 (1968).

23. R. W. Knighton and X.-R. Huang, "Linear birefringence of the central human cornea," Invest. Ophthalmol. Visual Sci. 43(1), 82-86 (2002).

24. S. Guido and R. T. Tranquillo, "A methodology for the systematic and quantitative study of cell contact guidance in oriented collagen gels. Correlation of fibroblast orientation and gel birefringence," J. Cell. Sci. 105(2), 317-331 (1993).

25. W. Kaminsky et al., "Simultaneous false-colour imaging of birefringence, extinction and transmittance at camera speed," J. Microsc. 228(2), 153-164 (2007).

26. S. Ross et al., "Quantitative image analysis of birefringent biological material," J. Microsc. 187(1), 62-67 (1997).

27. D. J. Maitland and J. T. Walsh, "Quantitative measurements of linear birefringence during heating of native collagen," Lasers Surg. Med. 20(3), 310-318 (1997).

28. J. de Boer et al., "Imaging thermally damaged tissue by polarization sensitive optical coherence tomography," Opt. Express 3(6), 212-218 (1998).

29. M. C. Pierce et al., "Collagen denaturation can be quantified in burned human skin using polarization-sensitive optical coherence tomography," Burns 30(6), 511-517 (2004).

30. Y. Yasuno et al., "Birefringence imaging of human skin by polarizationsensitive spectral interferometric optical coherence tomography," Opt. Lett. 27(20), 1803-1805 (2002).

31. M. Yamanari et al., "Fiber-based polarization-sensitive OCT for birefringence imaging of the anterior eye segment," Biomed. Opt. Express 6(2), 369-389 (2015).

32. G. Yao and L. V. Wang, "Two-dimensional depth-resolved Mueller matrix characterization of biological tissue by optical coherence tomography," Opt. Lett. 24(8), 537-539 (1999).

33. J. F. de Boer and T. E. Milner, "Review of polarization sensitive optical coherence tomography and Stokes vector determination," J. Biomed. Opt. 7(3), 359-371 (2002).

34. N. Ugryumova et al., "The collagen structure of equine articular cartilage, characterized using polarization-sensitive optical coherence tomography," J. Phys. D: Appl. Phys. 38(15), 2612-2619 (2005).

35. M.H. Smith et al., "Mueller matrix imaging polarimetry in dermatology," Proc. SPIE 3991, 210-216 (2000).

36. M.H. Smith, "Interpreting Mueller matrix images of tissues," Proc. SPIE 4257, 82-89 (2001).

37. J. M. Bueno and J. Jaronski, "Spatially resolved polarization properties for in vitro corneas," Ophthalmic. Physiol. Opt. 21(5), 384-392 (2001).

38. N. Ghosh et al., "Mueller matrix decomposition for polarized light assessment of biological tissues," J. Biophotonics 2(3), 145-156 (2009).

39. N. Ghosh et al., "Mueller matrix polarimetry for the characterization of complex random medium like biological tissues," Pramana 75(6), 1071-1086 (2010).

40. A. Pierangelo et al., "Multispectral Mueller polarimetric imaging detecting residual cancer and cancer regression after neoadjuvant treatment for colorectal carcinomas," J. Biomed. Opt. 18(4), 046014 (2013).

41. T. Novikova et al., "Polarimetric imaging for cancer diagnosis and staging," Opt. Photonics News 23(10), 26-33 (2012).

42. G. J. Van Blokland and S. C. Verhelst, "Corneal polarization in the living human eye explained with a biaxial model," JOSA A 4(1), 8290 (1987).

43. Y. P. Sinichkin et al., "The effect of the optical anisotropy of scattering media on the polarization state of scattered light," Opt. Spectrosc. 101(5), 802-810 (2006).

44. Y. P. Sinichkin, A. V. Spivak, and D. A. Yakovlev, "Simple parametric representations of the polarization optical properties of birefringent biological tissues in reflection polarization spectroscopy," Opt. Spectrosc. 107(6), 873-883 (2006).

45. V. Backman et al., "Polarized light scattering spectroscopy for quantitative measurement of epithelial cellular structures in situ," IEEE J. Sel. Top. Quant. Electron. 5(4), 1019-1026 (1999).

46. S. L. Jacques, J. C. Ramella-Roman, and K. Lee, "Imaging skin pathology with polarized light," J. Biomed. Opt. 7(3), 329-340 (2002).

47. J. C. Ramella-Roman et al., "Design, testing, and clinical studies of a handheld polarized light camera," J. Biomed. Opt. 9(6), 13051310 (2004)

48. O. V. Angelsky et al., "Polarization visualization and selection of biotissue image two-layer scattering medium," J. Biomed. Opt. 10(1), 014010 (2005).

49. M. F. Wood, X. Guo, and I. A. Vitkin, "Polarized light propagation in multiply scattering media exhibiting both linear birefringence and optical activity: Monte Carlo model and experimental methodology," J. Biomed. Opt. 12(1), 014029 (2007).

50. M. J. Girard et al., "Quantitative mapping of scleral fiber orientation in normal rat eyes," Invest. Ophthalmol. Visual Sci. 52(13), 9684$9693(2011)$

51. Y. Komai and T. Ushiki, "The three-dimensional organization of collagen fibrils in the human cornea and sclera," Invest. Ophthalmol. Visual Sci. 32(8), 2244-2258 (1991).

52. A. Thale, B. Tillmann, and R. Rochels, "Scanning electron-microscopic studies of the collagen architecture of the human sclera-normal and pathological findings," Ophthalmologica 210(3), 137-141 (1996).

53. S. L. Jacques, "Origins of tissue optical properties in the UVA, visible, and NIR regions," OSA TOPS Adv. Opt. Imaging Photon Migration 2, 364-369 (1996).

54. S. Nickell et al., "Anisotropy of light propagation in human skin," Phys. Med. Biol. 45(10), 2873-2886 (2000).

55. Y. E. Yarker, R. M. Aspden, and D. W. L. Hukins, "Birefringence of articular cartilage and the distribution of collagen fibril orientations," Connect. Tissue Res. 11(2-3), 207-213 (1983).

56. M. Aldrovani, A. M. A. Guaraldo, and B. C. Vidal, "Optical anisotropies in corneal stroma collagen fibers from diabetic spontaneous mice," Vision Res. 47(26), 3229-3237 (2007).

57. D. A. Yakovlev and V. G. Chigrinov, "A robust polarization-spectral method for determination of twisted liquid crystal layer parameters," J. Appl. Phys. 102(2), 023510 (2007).

58. D. A. Yakovlev, V. G. Chigrinov, and H.-S. Kwok, Modeling and Optimization of LCD Optical Performance, John Wiley \& Sons Ltd, Chichester, U.K. (2015).

59. A. B. Pravdin, V. A. Spivak, and D. A. Yakovlev, "On the possibility of noninvasive polarimetric determination of glucose content in skin," Opt. Spectrosc. 120(1), 45-49 (2016).

60. H. K. Aben, "Optical phenomena in photoelastic models by the rotation of principal axes," Exp. Mech. 6(1), 13-22 (1966).

61. H. K. Aben, "Characteristic directions in optics of twisted birefringent media," J. Opt. Soc. Am. A 3(9), 1414-1421 (1986).

62. P. Allia, C. Oldano, and L. Trossi, "Polarization transfer matrix for the transmission of light through liquid-crystal slabs," J. Opt. Soc. Am. B 5(12), 2452-2461 (1988).

63. D. A. Yakovlev, "Calculation of transmission characteristics of smoothly inhomogeneous anisotropic media in the approximation of negligible smallness of the bulk reflection: III. Analytical solutions," Opt. Spectrosc. 95(6), 944-951 (2003).

64. A. V. Spivak, "Examination of the effect of optical anisotropy of scattering media on polarization properties of the scattered light," PhD Thesis, Saratov State University, Saratov, Russia (2009). 
Dmitry D. Yakovlev received his MS degree from Saratov State University, Saratov, Russia. He is currently a PhD student at the Optics and Biophotonics Department at Saratov State University. His current research interests include polarized-light microscopy, photonics, optics of anisotropic media, and optics of biological tissues.

Marina E. Shvachkina received her MS degree in the Faculty of Physics from Saratov State University. Currently, she is a PhD student at the Optics and Biophotonics Department at Saratov State University. Her research interests include mechanical properties of collagenous biotissue, UVA-riboflavin collagen crosslinking of sclera, tissue optical clearing, and characterization of collagen-containing tissues by methods of polarization microscopy.

Maria M. Sherman received her PhD in optics from Saratov State University in 2009. Her current research interests include polarized-light microscopy, optics of inhomogeneous media, and biomedical optics.
Andrey V. Spivak received his $\mathrm{PhD}$ in biophysics from Saratov State University in 2009. His current research interests include biophysics and optics of biological tissues.

Alexander B. Pravdin graduated from Chemistry Department of Moscow State University in 1979. In 1990, he received the Candidate of Sciences (PhD) in physical chemistry from Moscow State University. Since 1988, he has been working at the Optics Department (later-Optics and Biophotonics Department) of Saratov State University, Saratov, Russia, where he is currently an associate professor. He lectures to students specializing in the field of physics of living systems and biological and medical photonics.

Dmitry A. Yakovlev is a senior researcher at the Education and Research Institute of Optics and Biophotonics, Saratov State University. He received his MS degree in physics and PhD in optics from Saratov State University in 1985 and 1998, respectively. He is the author of more than 40 journal papers and one book. His current research interests include optics of anisotropic media, biomedical optics, polarization optics, microscopy, and physics of liquid crystals. 